\title{
Power Laws on Weighted Branching Trees
}

Predrag R. Jelenković and Mariana Olvera-Cravioto

Abstract Consider distributional fixed-point equations of the form

$$
R \stackrel{\mathscr{D}}{=} f\left(Q, C_{i}, R_{i}, 1 \leq i \leq N\right),
$$

where $f(\cdot)$ is a possibly random real-valued function, $N \in\{0,1,2,3, \ldots\} \cup\{\infty\}$, $\left\{C_{i}\right\}_{i \in \mathbb{N}}$ are real-valued random weights and $\left\{R_{i}\right\}_{i \in \mathbb{N}}$ are iid copies of $R$, independent of $\left(Q, N, C_{1}, C_{2}, \ldots\right) ; \stackrel{\mathscr{D}}{=}$ represents equality in distribution. Fixed-point equations of this type are important for solving many applied probability problems, ranging from the average case analysis of algorithms to statistical physics. In this paper we present some of our recent work from [26, 27, 28, 36] that studies the power tail asymptotics of such solutions. We exemplify our techniques primarily on the nonhomogeneous equation, $R \stackrel{\mathscr{D}}{=} \sum_{i=1}^{N} C_{i} R_{i}+Q$, for which the power tail of the solution, $P(R>t)$, can be determined by three different factors: the multiplicative effect of the weights $C_{i}$; the sum of the weights $\sum C_{i}$; and the innovation variable $Q$.

\section{Introduction}

Our recent work on the analysis of recursions on weighted branching trees is motivated by the study of the nonhomogeneous linear fixed-point equation

$$
R \stackrel{\mathscr{D}}{=} \sum_{i=1}^{N} C_{i} R_{i}+Q
$$

Predrag R. Jelenković

Columbia University, Department of Electrical Engineering, e-mail: predrag@ee.columbia.edu

Mariana Olvera-Cravioto

Columbia University, Department of Industrial Engineering and Operations Research, e-mail: molvera@ieor.columbia.edu 
where $\left(Q, N, C_{1}, C_{2}, \ldots\right)$ is a real-valued random vector with $N \in \mathbb{N} \cup\{\infty\}, \mathbb{N}=$ $\{0,1,2,3, \ldots\}, P(|Q|>0)>0$, and $\left\{R_{i}\right\}_{i \in \mathbb{N}}$ is a sequence of iid random variables independent of $\left(Q, N, C_{1}, C_{2}, \ldots\right)$ having the same distribution as $R$. This recursion has been proposed as a stochastic approximation of Google's PageRank algorithm and possibly other ranking schemes of large information sets, e.g., the World Wide Web (WWW); see [46, 47, 26] and the references therein. There it is argued that the stochastic approach is helpful in understanding the qualitative behavior of PageRank given the large scale nature of the WWW. These types of weighted recursions, also studied in the literature on weighted branching processes [42] and branching random walks [10], are found in the probabilistic analysis of other algorithms as well [43, $40,1]$. The homogeneous $(Q \equiv 0)$ version of (1) has been studied extensively in the literature of weighted branching processes and multiplicative cascades, see $[2,4$, $24,34,33,30,10,23,17]$ and the references therein.

We now give some more details on the PageRank motivation that was mentioned above. PageRank assigns to each page a numerical weight that measures its relative importance with respect to other pages. We think of the Web as a very large interconnected graph where nodes correspond to pages. The Google trademarked algorithm PageRank defines the page rank as:

$$
R\left(p_{i}\right)=\frac{1-d}{n}+d \sum_{p_{j} \in M\left(p_{i}\right)} \frac{R\left(p_{j}\right)}{L\left(p_{j}\right)},
$$

where, using Google's notation, $p_{1}, p_{2}, \ldots, p_{n}$ are the pages under consideration, $M\left(p_{i}\right)$ is the set of pages that link to $p_{i}, L\left(p_{j}\right)$ is the number of outbound links on page $p_{j}, n$ is the total number of pages on the Web, and $d$ is a damping factor, usually $d=0.85$. While in principle the solution to (2) reduces to the solution of a large system (possibly billions) of linear equations, we believe that finding page ranks in such a way is unlikely to be insightful.

In particular, the division by the out-degree, $L\left(p_{j}\right)$ in equation (2), was meant to decrease the contribution of pages with highly inflated referencing, i.e., those pages that basically point/reference possibly indiscriminately to other documents. However, the stochastic approach reveals that highly ranked pages are essentially insensitive to the parameters of the out-degree distribution, and high ranks most likely occur either due to a pointer by a very highly ranked neighbor, or by pointers of a very large number of neighbors. Hence, PageRank may not reduce the effects of overly inflated referencing.

A stochastic approach to analyze (2) is to multiply it by $n$ and consider a typical node on the graph

$$
R \stackrel{\mathscr{D}}{=}(1-d)+d \sum_{i=1}^{N} \frac{R_{i}}{D_{i}},
$$

where $d>0, d E[1 / D]<1, N$ is a random variable independent of the $R_{i}$ 's and $D_{i}$ 's, the $D_{i}$ 's are iid random variables satisfying $D_{i} \geq 1$, and the $R_{i}$ 's are iid random variables having the same distribution as $R$. In terms of recursion (2), $R$ is the scale-free rank of a random page, $N$ corresponds to the in-degree of that node, the $R_{i}$ 's are 
the ranks of the pages pointing to it, and the $D_{i}$ 's correspond to the out-degrees of each of these pages. The experimental justification of these independence assumptions can be found in [45]. This stochastic setup was first introduced in [46], where the process resulting after a finite number of iterations of (3) was analyzed. Further generalization of (3) leads to (1), which was recently analyzed in $[47,26]$.

Furthermore, in computer science, a well known divide-and-conquer paradigm is used for designing efficient algorithms, where a problem is recursively divided into two or more sub-problems, until the sub-problems become simple enough to be solved directly. Such approach naturally leads to a recursive analysis, which in the case of randomized algorithms, often results in stochastic recursions of the type in (1). Among these, the most widely analyzed algorithm is Quicksort, whose analysis, after an appropriate normalization introduced in [41], reduces to the stochastic fixed point equation

$$
R \stackrel{\mathscr{D}}{=} U R_{1}+(1-U) R_{2}+Q,
$$

where $U$ is uniform, $Q=Q(U),\left\{R_{1}, R_{2}\right\}$ are independent copies of $R$ and independent of $(U, Q)$; for recent work see [18, 39] and the references therein. Similar binary equation also appear in the analysis of sequential absorption (packing) problems on a line, see equation (19) in [9]. Such problems are used for modeling memory fragmentation, advance reservation, particle absorption, e.g., see [15] and the references therein. Multidimensional versions of the fixed-point equation (1) have been considered in $[39,40]$ and more recently in [13].

In general, many applied probability problems, appearing in the average case analysis of algorithms and statistical physics, reduce to distributional fixed-point equations of the form

$$
R \stackrel{\mathscr{D}}{=} f\left(Q, C_{i}, R_{i}, 1 \leq i<N+1\right),
$$

where $f(\cdot)$ is a possibly random real-valued function, $N \in \mathbb{N} \cup\{\infty\}$, the $\left\{C_{i}\right\}_{i \in \mathbb{N}}$ are real-valued random weights and $\left\{R_{i}\right\}_{i \in \mathbb{N}}$ are iid copies of $R$, independent of $\left(Q, N, C_{1}, C_{2}, \ldots\right)$. For example, as discussed in [27], one can study the following distributional equations

$$
R \stackrel{\mathscr{D}}{=}\left(\bigvee_{i=1}^{N} C_{i} R_{i}\right) \vee Q, \quad R \stackrel{\mathscr{D}}{=}\left(\bigvee_{i=1}^{N} C_{i} R_{i}\right)+Q, \quad R \stackrel{\mathscr{D}}{=}\left(\sum_{i=1}^{N} C_{i} R_{i}\right) \vee Q .
$$

The solutions to equations of this type can be recursively constructed on a weighted branching tree, where $N$ represents the generic branching variable and the $\left\{C_{i}\right\}_{i \in \mathbb{N}}$ are the branching weights. For this reason, we also refer to (4) as recursions on weighted branching trees. The maximum recursion, the first one in (5), was previously studied in [7] under the assumption that $Q \equiv 0, N=\infty$, and the $\left\{C_{i}\right\}$ are realvalued deterministic constants, and the case of $Q \equiv 0$ and $\left\{C_{i}\right\} \geq 0$ being random was studied earlier in [25]. Furthermore, these max-plus type stochastic recursions appear in a wide variety of applications, see [1] for a recent survey. 
Special cases of the preceding recursions are important in many applied probability areas. For example, selecting $N=1$ in (1) yields the fixed-point equation satisfied by the first order autoregressive process. When $Q=1, C_{i} \equiv 1$, the steady state solution to (1) represents the total number of individuals born in an ordinary branching process. Similarly, by setting $N=Q \equiv 1$ and $X_{i}=\log C_{i}$ in the first equation in (5), one obtains the well studied supremum of a random walk and in particular the waiting time in the GI/GI/1 queue. By choosing the distributions of $N, Q$ or $C_{i}$ appropriately, all of these recursions can lead to heavy-tailed solutions.

In this paper we present some of our recent work from [26, 27, 28, 36] that studies the power-tail asymptotics of the solution $R$ to the preceding distributional fixed-point equations; all the omitted proofs can be found in these references. We will exemplify our techniques primarily on the nonhomogeneous equation (1), for which the tail of the solution, $P(R>t)$, can be determined by three different factors: the multiplicative effect of the weights $C_{i}$; the sum of the weights $\Sigma C_{i}$; and the innovation variable $Q$. In addition, to simplify the exposition, we only present the results for the case where $\left(Q, N, C_{1}, C_{2}, \ldots\right)$ is nonnegative and, when appropriate, we comment on the corresponding real-valued extensions.

First, we study the multiplicative effect of the weights by extending the implicit renewal theory of Goldie [19], which was derived for equations of the form

$R \stackrel{\mathscr{Q}}{=} f(Q, C, R)$ (equivalently $N \equiv 1$ in our case), to cover recursions on weighted branching trees. The extension of Goldie's theorem is presented in Theorem 1 of Section 3, and it enables the characterization of the power-tail behavior of the solutions $R$ to many equations of the form in (4), e.g., those stated in (1) and (5). One of the observations that allows this extension is that an appropriately constructed measure on a weighted branching tree is a renewal measure, see Lemma 1.

Then, in Section 4, we develop the necessary large deviations techniques that will enable us to study the tail behavior of $P(R>t)$ when it is determined by the sum of the weights, $\sum C_{i}$, or the innovation variable $Q$. The key technical contribution is the derivation of uniform bounds (in $n$ and $x$ ) for the distribution of the sum of the weights in the $n$th generation of a weighted branching tree, $P\left(W_{n}>x\right)$, given in Propositions 1 and 2. These uniform bounds are used to establish the geometric rate of convergence of the iterations of the fixed-point equation (1) to the solution $R$ constructed in Section 5.

Next, we exemplify the techniques we have developed on the nonhomogeneous linear recursion (1). In this regard, in Section 5, we first construct an explicit solution (13) to (1) on a weighted branching tree and then provide sufficient conditions for the finiteness of moments and the uniqueness of this solution under iterations in Lemmas 5 and 6, respectively. However, the fixed-point equation (1) can have additional stable solutions that do not satisfy Lemma 6 , as it was recently discovered in [5]. Earlier work for the case when $\left\{C_{i}\right\}, Q$ are deterministic real-valued constants can be found in $[42,6]$. Furthermore, it is worth noting that our moment estimates are explicit, see Lemma 4, which may be of independent interest. Our first main result about the constructed solution $R$ to (1) is given in Theorem 2, where through the extension of the Implicit Renewal Theorem it is shown that the multiplicative nature of the weights can lead to a power-tail behavior. Informally, our result shows, 
under some moment conditions, that

$$
P(R>x) \sim \frac{H}{x^{\alpha}} \quad \text { as } x \rightarrow \infty,
$$

where $\alpha$ is a solution to $E\left[\sum_{i=1}^{N} C_{i}^{\alpha}\right]=1$. In addition, for integer power exponent $(\alpha \in\{1,2,3, \ldots\})$ the constant $H$ can be explicitly computed as stated in Corollary 1. Furthermore, for non integer $\alpha$, we will explain how Lemma 2 can be used to obtain an explicit bound on $H$.

When the conditions for the Implicit Renewal Theorem fail, the tail behavior of $R$ can be determined by $P\left(\sum_{i=1}^{N} C_{i}>x\right)$ or $P(Q>x)$. Using our work on the large deviations of weighted random sums we give the corresponding results in Theorems 3 and 4, respectively. In particular, it is shown that if $P\left(\sum_{i=1}^{N} C_{i}>x\right)$ or $P(Q>x)$, are regularly varying with index $\alpha>1$, and certain moment conditions are satisfied, then, respectively,

$$
P(R>x) \sim H_{S} P\left(\sum_{i=1}^{N} C_{i}>x\right) \quad \text { or } \quad P(R>x) \sim H_{Q} P(Q>x)
$$

as $x \rightarrow \infty$, for some explicit constants $H_{S}, H_{Q}>0$. Lastly, we point out that we focus here only on the heavy-tailed solutions to (1), but it is known that (1) can also have light-tailed solutions, see [20] for the $N \equiv 1$ case and the discussion after Theorem 2.2 in [34] for the general branching case.

We conclude the paper with a brief analysis of other non-linear recursions, e.g., those stated in (5), that could be studied using the extension of the Implicit Renewal Theorem. The main difficulty in applying Theorem 1 is in verifying the conditions of the theorem for a specific fixed-point equation. In this regard, we argue that the two technical lemmas, Lemmas 7 and 8, can be helpful for this purpose.

The rest of the paper is organized as follows. Section 2 contains the construction of the weighted branching tree. In Section 3 we present the extension of the implicit renewal theorem to trees and, in Section 4, we derive the uniform large deviation bounds for $P\left(W_{n}>x\right)$. Section 5 exemplifies our techniques on the nonhomogeneous linear equation (1), and Section 6 briefly discusses how the developed tools can be applied to other fixed-point equations, e.g., those in (5).

\section{Weighted branching tree}

First we construct a random tree $\mathscr{T}$. We use the notation $\emptyset$ to denote the root node of $\mathscr{T}$, and $A_{n}, n \geq 0$, to denote the set of all individuals in the $n$th generation of $\mathscr{T}, A_{0}=\{\emptyset\}$. Let $Z_{n}$ be the number of individuals in the $n$th generation, that is, $Z_{n}=\left|A_{n}\right|$, where $|\cdot|$ denotes the cardinality of a set; in particular, $Z_{0}=1$.

Next, let $\mathbb{N}_{+}=\{1,2,3, \ldots\}$ be the set of positive integers and let $U=\bigcup_{k=0}^{\infty}\left(\mathbb{N}_{+}\right)^{k}$ be the set of all finite sequences $\mathbf{i}=\left(i_{1}, i_{2}, \ldots, i_{n}\right)$, where by convention $\mathbb{N}_{+}^{0}=$ $\{\emptyset\}$ contains the null sequence $\emptyset$. To ease the exposition, for a sequence $\mathbf{i}=$ 


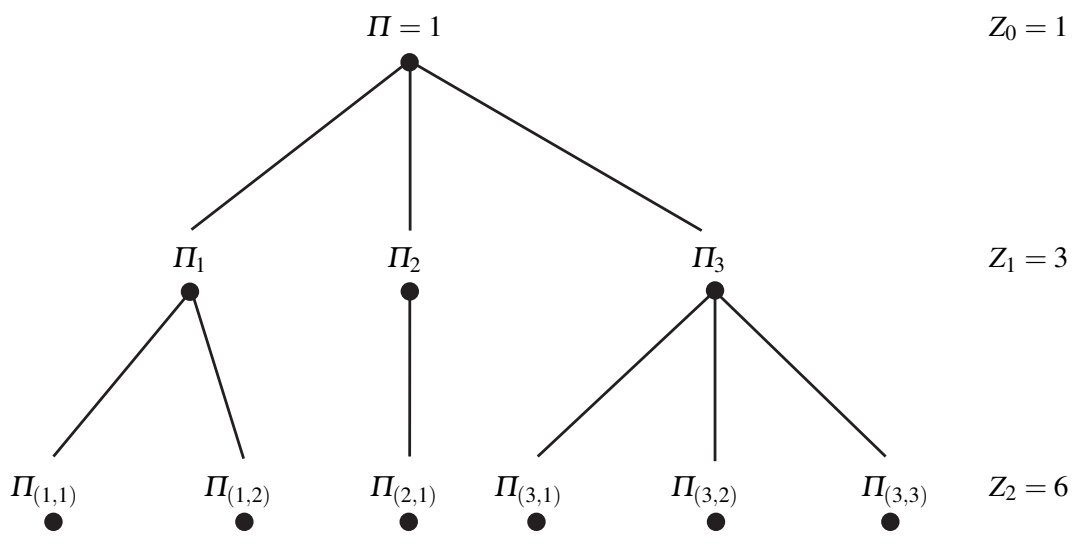

Fig. 1 Weighted branching tree

$\left(i_{1}, i_{2}, \ldots, i_{k}\right) \in U$ we write $\mathbf{i} \mid n=\left(i_{1}, i_{2}, \ldots, i_{n}\right)$, provided $k \geq n$, and $\mathbf{i} \mid 0=\emptyset$ to denote the index truncation at level $n, n \geq 0$. Also, for $\mathbf{i} \in A_{1}$ we simply use the notation $\mathbf{i}=i_{1}$, that is, without the parenthesis. Similarly, for $\mathbf{i}=\left(i_{1}, \ldots, i_{n}\right)$ we will use $(\mathbf{i}, j)=\left(i_{1}, \ldots, i_{n}, j\right)$ to denote the index concatenation operation, if $\mathbf{i}=\emptyset$, then $(\mathbf{i}, j)=j$.

We iteratively construct the tree as follows. Let $N$ be the number of individuals born to the root node $\emptyset, N_{\emptyset}=N$, and let $\left\{N_{\mathbf{i}}\right\}_{\mathbf{i} \in U, \mathbf{i} \neq \emptyset}$ be iid copies of $N$. Define now

$$
A_{1}=\{i \in \mathbb{N}: 1 \leq i \leq N\}, \quad A_{n}=\left\{\left(\mathbf{i}, i_{n}\right) \in U: \mathbf{i} \in A_{n-1}, 1 \leq i_{n} \leq N_{\mathbf{i}}\right\} .
$$

It follows that the number of individuals $Z_{n}=\left|A_{n}\right|$ in the $n$th generation, $n \geq 1$, satisfies the branching recursion

$$
Z_{n}=\sum_{\mathbf{i} \in A_{n-1}} N_{\mathbf{i}}
$$

Now, we construct the weighted branching tree $\mathscr{T}_{Q, C}$ as follows. We start by assigning the vector $\left(Q_{\emptyset}, N_{\emptyset}, C_{(\emptyset, 1)}, C_{(\emptyset, 2)}, \ldots\right) \equiv\left(Q, N, C_{1}, C_{2}, \ldots\right)$ to the root node $\emptyset$. Next, let $\left\{\left(Q_{\mathbf{i}}, N_{\mathbf{i}}, C_{(\mathbf{i}, 1)}, C_{(\mathbf{i}, 2)}, \ldots\right)\right\}_{\mathbf{i} \in U, \mathbf{i} \neq \emptyset}$ be a sequence of iid copies of $\left(Q, N, C_{1}, C_{2}, \ldots\right)$. Recall that $N_{\emptyset}$ determines the number of nodes in the first generation of $\mathscr{T}$ according to (6), and assign to each node in the first generation its corresponding vector $\left(Q_{i}, N_{i}, C_{(i, 1)}, C_{(i, 2)}, \ldots\right)$ from the preceding iid sequence. In general, for $n \geq 2$, to each node $\mathbf{i} \in A_{n-1}$ we assign its corresponding vector $\left(Q_{\mathbf{i}}, N_{\mathbf{i}}, C_{(\mathbf{i}, 1)}, C_{(\mathbf{i}, 2)}, \ldots\right)$ from the sequence and construct $A_{n}=\left\{\left(\mathbf{i}, i_{n}\right) \in U: \mathbf{i} \in\right.$ $\left.A_{n-1}, 1 \leq i_{n} \leq N_{\mathbf{i}}\right\}$. For each node in $\mathscr{T}_{Q, C}$ we also define the weight $\Pi_{\left(i_{1}, \ldots, i_{n}\right)}$ via the recursion

$$
\Pi_{i_{1}}=C_{i_{1}}, \quad \Pi_{\left(i_{1}, \ldots, i_{n}\right)}=C_{\left(i_{1}, \ldots, i_{n}\right)} \Pi_{\left(i_{1}, \ldots, i_{n-1}\right)}, \quad n \geq 2,
$$


where $\Pi=1$ is the weight of the root node. Note that the weight $\Pi_{\left(i_{1}, \ldots, i_{n}\right)}$ is equal to the product of all the weights $C_{(\cdot)}$ along the branch leading to node $\left(i_{1}, \ldots, i_{n}\right)$, as depicted in Figure 1. In some places, e.g., in the following section, the value of $Q$ may be of no importance, and thus we will consider a weighted branching tree defined by the smaller vector $\left(N, C_{1}, C_{2}, \ldots\right)$. This tree can be obtained from $\mathscr{T}_{Q, C}$ by simply disregarding the values for $Q_{(\cdot)}$ and is denoted by $\mathscr{T}_{C}$.

The objective of this paper is to present a variety of results that analyze recursions and fixed-point equations embedded in this weighted branching tree.

\section{Implicit renewal theorem on trees}

In this section we present an extension of Goldie's Implicit Renewal Theorem [19] to weighted branching trees. The observation that facilitates this generalization is the following lemma which shows that a certain measure on a tree is actually a product measure; a similar measure was used in a different context in [11]. Throughout the paper we use the standard convention $0^{\alpha} \log 0=0$ for all $\alpha>0$, and the notation $x^{+}=\max \{x, 0\}, x^{-}=-\min \{x, 0\}$.

Lemma 1. Let $\mathscr{T}_{C}$ be the weighted branching tree defined by the nonnegative vector $\left(N, C_{1}, C_{2}, \ldots\right)$, where $N \in \mathbb{N} \cup\{\infty\}$. For any $n \in \mathbb{N}$ and $\mathbf{i} \in A_{n}$, let $V_{\mathbf{i}}=\log \Pi_{\mathbf{i}}$. For $\alpha>0$ define the measure

$$
\mu_{n}(d t)=e^{\alpha t} E\left[\sum_{\mathbf{i} \in A_{n}} 1\left(V_{\mathbf{i}} \in d t\right)\right], \quad n=1,2, \ldots,
$$

and let $\eta(d t)=\mu_{1}(d t)$. Suppose that there exists $j \geq 1$ with $P\left(N \geq j, C_{j}>\right.$ $0)>0$ such that the measure $P\left(\log C_{j} \in d u, C_{j}>0, N \geq j\right)$ is nonarithmetic, $E\left[\sum_{i=1}^{N} C_{i}^{\gamma}\right]<\infty$ for some $0 \leq \gamma<\alpha$, and $E\left[\sum_{i=1}^{N} C_{i}^{\alpha}\right]=1$. Then, $\eta(\cdot)$ is a nonarithmetic probability measure on $\mathbb{R}$ that places no mass at $-\infty$ and has mean

$$
\mu \triangleq \int_{-\infty}^{\infty} u \eta(d u)=E\left[\sum_{j=1}^{N} C_{j}^{\alpha} \log C_{j}\right] .
$$

Furthermore, $\mu_{n}(d t)=\eta^{* n}(d t)$, where $\eta^{* n}$ denotes the nth convolution of $\eta$ with itself.

Note that $E\left[\sum_{i=1}^{N} C_{i}^{\gamma}\right]<\infty$ and $E\left[\sum_{i=1}^{N} C_{i}^{\alpha}\right]<\infty$ for $0 \leq \gamma<\alpha$ implies $E\left[\sum_{i=1}^{N} C_{i}^{\alpha}\left(\log C_{i}\right)^{-}\right]<\infty$, and therefore the mean of $\eta(\cdot)$ is well defined.

We now present Theorem 3.1 of [27], which is a generalization of Goldie's Implicit Renewal Theorem [19] that enables the analysis of recursions on weighted branching trees. Note that except for the independence assumption, the random variable $R$ and the vector $\left(N, C_{1}, C_{2}, \ldots\right)$ are arbitrary, and therefore the applicability of this theorem goes beyond the recursions that we study here. When this theorem is 
applied to specific recursions, one can use the nature of the recursion to verify the conditions of the theorem. Typically, it is the absolute integrability in (7) that requires the most work. Throughout the paper we use $g(x) \sim f(x)$ as $x \rightarrow \infty$ to denote $\lim _{x \rightarrow \infty} g(x) / f(x)=1$.

Theorem 1. Let $\left(N, C_{1}, C_{2}, \ldots\right)$ be a nonnegative random vector, where $N \in \mathbb{N} \cup$ $\{\infty\}$. Suppose that there exists $j \geq 1$ with $P\left(N \geq j, C_{j}>0\right)>0$ such that the measure $P\left(\log C_{j} \in d u, C_{j}>0, N \geq j\right)$ is nonarithmetic. Assume further that $0<E\left[\sum_{j=1}^{N} C_{j}^{\alpha} \log C_{j}\right]<\infty, E\left[\sum_{j=1}^{N} C_{j}^{\alpha}\right]=1, E\left[\sum_{j=1}^{N} C_{j}^{\gamma}\right]<\infty$ for some $0 \leq$ $\gamma<\alpha$, and that $R \geq 0$ is independent of $\left(N, C_{1}, C_{2}, \ldots\right)$ with $E\left[R^{\beta}\right]<\infty$ for any $0<\beta<\alpha$. If

$$
\int_{0}^{\infty}\left|P(R>t)-E\left[\sum_{j=1}^{N} 1\left(C_{j} R>t\right)\right]\right| t^{\alpha-1} d t<\infty,
$$

then

$$
P(R>t) \sim H t^{-\alpha}, \quad t \rightarrow \infty,
$$

where $0 \leq H<\infty$ is given by

$$
H=\frac{1}{E\left[\sum_{j=1}^{N} C_{j}^{\alpha} \log C_{j}\right]} \int_{0}^{\infty} v^{\alpha-1}\left(P(R>v)-E\left[\sum_{j=1}^{N} 1\left(C_{j} R>v\right)\right]\right) d v .
$$

Remark 1. (i) As pointed out in [19], the statement of the theorem only has content when $R$ has infinite moment of order $\alpha$, since otherwise the constant $H$ is zero. (ii) This theorem was recently generalized in Theorem 3.4 of [28] to incorporate realvalued weights $\left\{C_{i}\right\}$ and real-valued $R$. (iii) When the $\left\{\log C_{i}\right\}$ are lattice-valued, a similar version of the theorem was derived by using the corresponding Renewal Theorem for lattice random walks, see Theorem 3.7 in [28]. (iv) To see that the condition $E\left[\sum_{j=1}^{N} C_{j}^{\gamma}\right]<\infty$ for some $0 \leq \gamma<\alpha$ is needed, consider the following example. Fix $k \geq 2$ to be such that $A=\sum_{j=k}^{\infty} 1 /\left(j(\log j)^{3}\right)$ and $B=\sum_{j=k}^{\infty}(\log j+$ $3 \log \log j) /\left(j(\log j)^{3}\right)$ are both smaller than $1 / 2$, and choose $C=e^{X}$ where $X$ is exponentially distributed with mean $(1-A)$. Now set $C_{j}=C /\left(j(\log j)^{3}\right)$ for $j \geq k$ and $C_{j}=0$ otherwise $(N=\infty)$. Then, $E\left[\sum_{j=k}^{\infty} C_{j}\right]=1$ and $E\left[\sum_{j=k}^{\infty} C_{j} \log C_{j}\right]=$ $A^{-1}(1-A-B)>0$, but $E\left[\sum_{j=k}^{\infty} C_{j}^{\gamma}\right]=\infty$ for any $0 \leq \gamma<1$. (v) As noted in [19], the early ideas of applying renewal theory to study the power tail asymptotics of autoregressive processes (perpetuities) is due to [31] and [22].

Sketch of the proof of Theorem 1. Let $\mathscr{T}_{C}$ be the weighted branching tree defined by the nonnegative vector $\left(N, C_{1}, C_{2}, \ldots\right)$. For each $\mathbf{i} \in A_{n}$ and all $k \leq n$ define $V_{\mathbf{i} \mid k}=$ $\log \Pi_{\mathbf{i} \mid k}$; note that $\Pi_{\mathbf{i} \mid k}$ is independent of $N_{\mathbf{i} \mid k}$ but not of $N_{\mathbf{i} \mid s}$ for any $0 \leq s \leq k-1$. Also note that $\mathbf{i} \mid n=\mathbf{i}$ since $\mathbf{i} \in A_{n}$. Let $\mathscr{F}_{k}, k \geq 1$, denote the $\sigma$-algebra generated by $\left\{\left(N_{\mathbf{i}}, C_{(\mathbf{i}, 1)}, C_{(\mathbf{i}, 2)}, \ldots\right): \mathbf{i} \in A_{j}, 0 \leq j \leq k-1\right\}$, and let $\mathscr{F}_{0}=\sigma(\emptyset, \Omega), \Pi_{\mathbf{i} \mid 0} \equiv 1$. 
Assume also that $R$ is independent of the entire weighted tree, $\mathscr{T}_{C}$. Then, for any $t \in \mathbb{R}$, we can write $P\left(R>e^{t}\right)$ via a telescoping sum as follows

$$
\begin{aligned}
P & \left(R>e^{t}\right) \\
= & \sum_{k=0}^{n-1}\left(E\left[\sum_{(\mathbf{i} \mid k) \in A_{k}} 1\left(\Pi_{\mathbf{i} \mid k} R>e^{t}\right)\right]-E\left[\sum_{(\mathbf{i} \mid k+1) \in A_{k+1}} 1\left(\Pi_{\mathbf{i} \mid k+1} R>e^{t}\right)\right]\right) \\
& +E\left[\sum_{(\mathbf{i} \mid n) \in A_{n}} 1\left(\Pi_{\mathbf{i} \mid n} R>e^{t}\right)\right] \\
= & \sum_{k=0}^{n-1} E\left[\sum_{(\mathbf{i} \mid k) \in A_{k}}\left(1\left(\Pi_{\mathbf{i} \mid k} R>e^{t}\right)-\sum_{j=1}^{N_{\mathbf{i} \mid k}} 1\left(\Pi_{\mathbf{i} \mid k} C_{(\mathbf{i} \mid k, j)} R>e^{t}\right)\right)\right] \\
& +E\left[\sum_{(\mathbf{i} \mid n) \in A_{n}} 1\left(\Pi_{\mathbf{i} \mid n} R>e^{t}\right)\right] \\
= & \sum_{k=0}^{n-1} E\left[\sum_{(\mathbf{i} \mid k) \in A_{k}} E\left[1\left(R>e^{t-V_{\mathbf{i} \mid k}}\right)-\sum_{j=1}^{N_{\mathbf{i} \mid k}} 1\left(C_{(\mathbf{i} \mid k, j)} R>e^{\left.t-V_{\mathbf{i} \mid k}\right)} \mid \mathscr{F}_{k}\right]\right]\right. \\
& +E\left[\sum_{(\mathbf{i} \mid n) \in A_{n}} 1\left(\Pi_{\mathbf{i} \mid n} R>e^{t}\right)\right] .
\end{aligned}
$$

Now, define the measures $\mu_{n}$ according to Lemma 1 and let

$$
\begin{gathered}
v_{n}(d t)=\sum_{k=0}^{n} \mu_{k}(d t), \quad g(t)=e^{\alpha t}\left(P\left(R>e^{t}\right)-E\left[\sum_{j=1}^{N} 1\left(C_{j} R>e^{t}\right)\right]\right), \\
r(t)=e^{\alpha t} P\left(R>e^{t}\right) \quad \text { and } \quad \delta_{n}(t)=e^{\alpha t} E\left[\sum_{(\mathbf{i} \mid n) \in A_{n}} 1\left(\Pi_{\mathbf{i} \mid n} R>e^{t}\right)\right] .
\end{gathered}
$$

Recall that $R$ and $\left(N_{\mathbf{i} \mid k}, C_{(\mathbf{i} \mid k, 1)}, C_{(\mathbf{i} \mid k, 2)}, \ldots\right)$ are independent of $\mathscr{F}_{k}$, from where it follows that

$$
E\left[1\left(R>e^{t-V_{\mathbf{i} \mid k}}\right)-\sum_{j=1}^{N_{\mathbf{i} \mid k}} 1\left(C_{(\mathbf{i} \mid k, j)} R>e^{t-V_{\mathbf{i} \mid k}}\right) \mid \mathscr{F}_{k}\right]=e^{\alpha\left(V_{\mathbf{i} \mid k}-t\right)} g\left(t-V_{\mathbf{i} \mid k}\right) .
$$

Then, for any $t \in \mathbb{R}$ and $n \in \mathbb{N}$,

$$
r(t)=\sum_{k=0}^{n-1} E\left[\sum_{(\mathbf{i} \mid k) \in A_{k}} e^{\alpha V_{\mathbf{i} \mid k}} g\left(t-V_{\mathbf{i} \mid k}\right)\right]+\delta_{n}(t)=\left(g * v_{n-1}\right)(t)+\delta_{n}(t) .
$$

Next, using the assumptions of the theorem, one can show that $\delta_{n}(t) \rightarrow 0$ as $n \rightarrow \infty$, and furthermore,

$$
r(t)=g * v(t),
$$


where $v(d t)=\sum_{k=0}^{\infty} \eta^{* k}(d t)$; see $[27,28]$ for more details. Now, the result would follow from the key renewal theorem for two-sided random walks if it were not for the fact that $g$ is not necessarily directly Riemann integrable. To overcome this difficulty one can introduce a smoothing transform, similarly as it was done in [19], and apply the key renewal theorem to the transformed equation to show that

$$
e^{-t} \int_{0}^{e^{t}} v^{\alpha} P(R>v) d v \rightarrow H, \quad t \rightarrow \infty .
$$

Finally, by a version of the monotone density theorem (see Lemma 9.3 in [19]), one derives

$$
P(R>t) \sim H t^{-\alpha}, \quad t \rightarrow \infty,
$$

where

$$
\begin{aligned}
H & =\frac{1}{\mu} \int_{-\infty}^{\infty} g(t) d t \\
& =\frac{1}{\mu} \int_{0}^{\infty} v^{\alpha-1}\left(P(R>v)-E\left[\sum_{j=1}^{N} 1\left(C_{j} R>v\right)\right]\right) d v
\end{aligned}
$$

and $\mu$ was defined in Lemma 1 .

\section{Large deviations analysis}

In this section we give the main technical result that allows the analysis of the solutions to recursions on weighted branching trees when the conditions for the Implicit Renewal Theorem do not apply, but either the sum of the weights, $\sum_{i=1}^{N} C_{i}$, or the innovation, $Q$, has a heavy-tailed distribution. The analysis in these cases is based on a uniform bound for the tail distribution of the sum of the weights on the $n$th generation of a weighted branching tree, which we formally define below.

Let $\left\{W_{n}: n \geq 0\right\}$ be the process constructed on $\mathscr{T}_{Q, C}$ via

$$
W_{0}=Q, \quad W_{n}=\sum_{\mathbf{i} \in A_{n}} Q_{\mathbf{i}} \Pi_{\mathbf{i}}, \quad n \geq 1 .
$$

Since the tree structure repeats itself after the first generation, $W_{n}$ satisfies

$$
W_{n} \stackrel{\mathscr{P}}{=} \sum_{k=1}^{N} C_{k} W_{(n-1), k}
$$

where $\left\{W_{(n-1), k}\right\}$ is a sequence of iid random variables independent of $\left(N, C_{1}, C_{2}, \ldots\right)$ and having the same distribution as $W_{n-1}$. 
We now proceed to compute explicit moment bounds for $W_{n}$. The next lemma is the key to this analysis; a generalization to real-valued random variables can be found in [28].

Lemma 2. For any $k \in \mathbb{N} \cup\{\infty\}$ let $\left\{C_{i}\right\}_{i=1}^{k}$ be a sequence of nonnegative random variables and let $\left\{Y_{i}\right\}_{i=1}^{k}$ be a sequence of nonnegative iid random variables, independent of the $\left\{C_{i}\right\}$, having the same distribution as $Y$. For $\beta>1$ set $p=\lceil\beta\rceil \in\{2,3,4, \ldots\}$, and if $k=\infty$ assume that $\sum_{i=1}^{\infty} C_{i} Y_{i}<\infty$ a.s. Then,

$$
E\left[\left(\sum_{i=1}^{k} C_{i} Y_{i}\right)^{\beta}-\sum_{i=1}^{k}\left(C_{i} Y_{i}\right)^{\beta}\right] \leq\left(E\left[Y^{p-1}\right]\right)^{\beta /(p-1)} E\left[\left(\sum_{i=1}^{k} C_{i}\right)^{\beta}\right] .
$$

Remark 2. Note that the preceding lemma does not exclude the case when $E\left[\left(\sum_{i=1}^{k} C_{i} Y_{i}\right)^{\beta}\right]=\infty$ but $E\left[\left(\sum_{i=1}^{k} C_{i} Y_{i}\right)^{\beta}-\sum_{i=1}^{k}\left(C_{i} Y_{i}\right)^{\beta}\right]<\infty$.

We now give estimates for the $\beta$-moments of $W_{n}$ for $\beta \in(0,1]$ and $\beta>1$ in Lemmas 3 and 4, respectively; their proofs can be found in [27]. Throughout the rest of the paper define $\rho_{\beta}=E\left[\sum_{i=1}^{N} C_{i}^{\beta}\right]$ for any $\beta>0$, and $\rho \equiv \rho_{1}$.

Lemma 3. For $0<\beta \leq 1$ and all $n \geq 0$,

$$
E\left[W_{n}^{\beta}\right] \leq E\left[Q^{\beta}\right] \rho_{\beta}^{n} .
$$

Lemma 4. For $\beta>1$ suppose $E\left[Q^{\beta}\right]<\infty, E\left[\left(\sum_{i=1}^{N} C_{i}\right)^{\beta}\right]<\infty$, and $\rho \vee \rho_{\beta}<1$. Then, there exists a constant $K_{\beta}<\infty$ such that for all $n \geq 0$,

$$
E\left[W_{n}^{\beta}\right] \leq K_{\beta}\left(\rho \vee \rho_{\beta}\right)^{n} .
$$

The main technical result of this section provides a uniform bound (uniform in $n$ and $x)$ for $P\left(W_{n}>x\right)$ under the assumption that either $P\left(\sum_{i=1}^{N} C_{i}>x\right) \in \mathscr{R}_{-\alpha}$ or $P(Q>x) \in \mathscr{R}_{-\alpha}$, where $\mathscr{R}_{-\alpha}$ is the family of regularly varying functions with index $-\alpha$. For completeness we give the definition below.

Definition 1. A function $f$ is regularly varying at infinity with index $\lambda$, denoted $f \in \mathscr{R}_{\lambda}$, if $f(x)=x^{\lambda} L(x)$ for some slowly varying function $L$; and $L:[0, \infty) \rightarrow(0, \infty)$ is slowly varying if $\lim _{x \rightarrow \infty} L(t x) / L(x)=1$ for any $t>0$.

We now state the two main results of this section; their proofs are given in [36].

Proposition 1. Let $Z_{N}=\sum_{i=1}^{N} C_{i}$ and suppose $P\left(Z_{N}>x\right) \in \mathscr{R}_{-\alpha}$ with $\alpha>1$. Assume further that $E\left[Q^{\alpha+\varepsilon}\right]<\infty$ and $\rho_{\alpha+\varepsilon}<\infty$ for some $\varepsilon>0$. Fix $\rho \vee \rho_{\alpha}<\eta<1$. Then, there exists a finite constant $K=K(\eta, \varepsilon)>0$ such that for all $n \geq 1$ and all $x \geq 1$,

$$
P\left(W_{n}>x\right) \leq K \eta^{n} P\left(Z_{N}>x\right) .
$$


Remark 3. Note that we can easily obtain a weaker uniform bound by applying the moment estimate on $E\left[W_{n}^{\beta}\right]$ from Lemma 4, i.e., $P\left(W_{n}>x\right) \leq E\left[W_{n}^{\beta}\right] x^{-\beta} \leq K_{\beta}(\rho \vee$ $\left.\rho_{\beta}\right)^{n} x^{-\beta}$ for some $0<\beta<\alpha$, so the tradeoff in (10) is a slightly larger geometric term for a lighter tail distribution. However, the assertion in (10) is considerably more difficult to prove.

The corresponding result for the case when $P(Q>x) \in \mathscr{R}_{-\alpha}$ is given below.

Proposition 2. Suppose $P(Q>x) \in \mathscr{R}_{-\alpha}$, with $\alpha>1, E\left[Z_{N}^{\alpha+\varepsilon}\right]<\infty$ for some $\varepsilon>0$, and let $\rho \vee \rho_{\alpha}<\eta<1$. Then, there exists a finite constant $K=K(\eta, \varepsilon)>0$ such that for all $n \geq 1$ and all $x \geq 1$,

$$
P\left(W_{n}>x\right) \leq K \eta^{n} P(Q>x) .
$$

\section{The linear recursion $R \stackrel{\mathscr{D}}{=} \sum_{i=1}^{N} C_{i} R_{i}+Q$}

This section focuses on the analysis of the linear nonhomogeneous equation (1), and it is further divided into the three possible sources of power-law tails of the solution $R$. Before we proceed with the analysis, we give below an explicit construction of $R$ on the weighted branching tree $\mathscr{T}_{Q, C}$ and show that, under appropriate conditions, this solution is the unique limit under iterations of (1). Recall that throughout the paper we assume that the vector $\left(Q, N, C_{1}, C_{2}, \ldots\right)$ is nonnegative.

Define the process $\left\{R^{(n)}\right\}_{n \geq 0}$ according to

$$
R^{(n)}=\sum_{k=0}^{n} W_{k}, \quad n \geq 0,
$$

that is, $R^{(n)}$ is the sum of the weights of all the nodes on the tree up to the $n$th generation. It is not hard to see that $R^{(n)}$ satisfies the recursion

$$
R^{(n)}=\sum_{j=1}^{N_{\emptyset}} C_{(\emptyset, j)} R_{j}^{(n-1)}+Q_{\emptyset}=\sum_{j=1}^{N} C_{j} R_{j}^{(n-1)}+Q, \quad n \geq 1,
$$

where $\left\{R_{j}^{(n-1)}\right\}$ are independent copies of $R^{(n-1)}$ corresponding to the tree starting with individual $j$ in the first generation and ending on the $n$th generation; note that $R_{j}^{(0)}=Q_{j}$.

Next, define the random variable $R$ according to

$$
R \triangleq \lim _{n \rightarrow \infty} R^{(n)}=\sum_{k=0}^{\infty} W_{k}
$$

where the limit is properly defined by (11) and monotonicity. Hence, it is easy to verify, by applying monotone convergence in (12), that $R$ must solve 


$$
R=\sum_{j=1}^{N_{\emptyset}} C_{(\emptyset, j)} R_{j}^{(\infty)}+Q_{\emptyset}=\sum_{j=1}^{N} C_{j} R_{j}^{(\infty)}+Q
$$

where $\left\{R_{j}^{(\infty)}\right\}_{j \in \mathbb{N}}$ are iid, have the same distribution as $R$, and are independent of $\left(Q, N, C_{1}, C_{2}, \ldots\right)$.

The derivation provided above implies in particular the existence of a solution in distribution to (1). Moreover, under additional technical conditions, $R$ is the unique solution under iterations as it will be defined and shown in the following section. The constructed $R$, as defined in (13), is the main object of study in the remainder of this section. Note that, in view of the very recent work in [5], (1) may have other stable-law solutions that are not considered here. The lemma below gives sufficient conditions for the finiteness of moments of $R$, see [27] for a proof.

Lemma 5. Assume that $E\left[Q^{\beta}\right]<\infty$ for some $\beta>0$. In addition, suppose that either (i) $\rho_{\beta}<1$ if $0<\beta<1$, or (ii) $\left(\rho \vee \rho_{\beta}\right)<1$ and $E\left[\left(\sum_{i=1}^{N} C_{i}\right)^{\beta}\right]<\infty$ if $\beta \geq 1$. Then, $E\left[R^{\gamma}\right]<\infty$ for all $0<\gamma \leq \beta$, and in particular, $R<\infty$ a.s. Moreover, if $\beta \geq 1$, $R^{(n)} \stackrel{L_{\beta}}{\rightarrow} R$, where $L_{\beta}$ denotes $\beta$-norm convergence.

Remark 4. It is interesting to observe that for $\beta>1$ the conditions $\rho_{\beta}<1$ and $E\left[\left(\sum_{i=1}^{N} C_{i}\right)^{\beta}\right]<\infty$ are consistent with Theorem 3.1 in [4], Proposition 4 in [24] and Theorem 2.1 in [34], which give the conditions for the finiteness of the $\beta$-moment of the solution to the related critical $\left(\rho_{1}=1\right)$ homogeneous $(Q \equiv 0)$ equation.

Next, we show that under some technical conditions, the iteration of recursion (1) results in a process that converges in distribution to $R$ for any initial condition $R_{0}^{*}$. To this end, consider a weighted branching tree $\mathscr{T}_{Q, C}$, as defined in Section 2. Now, define

$$
R_{n}^{*} \triangleq R^{(n-1)}+W_{n}\left(R_{0}^{*}\right), \quad n \geq 1,
$$

where $R^{(n-1)}$ is given by (11),

$$
W_{n}\left(R_{0}^{*}\right)=\sum_{\mathbf{i} \in A_{n}} R_{0, \mathbf{i}}^{*} \Pi_{\mathbf{i}},
$$

and $\left\{R_{0, \mathbf{i}}^{*}\right\}_{\mathbf{i} \in U}$ are iid copies of an initial value $R_{0}^{*}$, independent of the entire weighted tree $\mathscr{T}_{Q, C}$. It follows from (12) and (14) that, for $n \geq 0$,

$$
\begin{aligned}
R_{n+1}^{*} & =\sum_{j=1}^{N} C_{j} R_{j}^{(n-1)}+Q+W_{n+1}\left(R_{0}^{*}\right) \\
& =\sum_{j=1}^{N} C_{j}\left(R_{j}^{(n-1)}+\sum_{\mathbf{i} \in A_{n, j}} R_{0, \mathbf{i}}^{*} \prod_{k=2}^{n} C_{\left(j, \ldots, i_{k}\right)}\right)+Q,
\end{aligned}
$$

where $\left\{R_{j}^{(n-1)}\right\}$ are independent copies of $R^{(n-1)}$ corresponding to the tree starting with individual $j$ in the first generation and ending on the $n$th generation, and $A_{n, j}$ 
is the set of all nodes in the $(n+1)$ th generation that are descendants of individual $j$ in the first generation. It follows that

$$
R_{n+1}^{*}=\sum_{j=1}^{N} C_{j} R_{n, j}^{*}+Q
$$

where $\left\{R_{n, j}^{*}\right\}$ are the expressions inside the parenthesis in (15). Clearly, $\left\{R_{n, j}^{*}\right\}$ are iid copies of $R_{n}^{*}$, thus we show that $R_{n}^{*}$ is equal in distribution to the process derived by iterating (1) with an initial condition $R_{0}^{*}$. The following lemma shows that $R_{n}^{*} \Rightarrow$ $R$ for any initial condition $R_{0}^{*}$ satisfying a moment assumption, where $\Rightarrow$ denotes convergence in distribution; see [27] for a proof.

Lemma 6. For any initial condition $R_{0}^{*} \geq 0$, if $E\left[Q^{\beta}\right], E\left[\left(R_{0}^{*}\right)^{\beta}\right]<\infty$ and $\rho_{\beta}=$ $E\left[\sum_{i=1}^{N} C_{i}^{\beta}\right]<1$ for some $0<\beta \leq 1$, then

$$
R_{n}^{*} \Rightarrow R
$$

with $E\left[R^{\beta}\right]<\infty$. Furthermore, under these assumptions, the distribution of $R$ is the unique solution with finite $\beta$-moment to recursion (1).

Remark 5. (i) Note that when $E[N]<1$ the branching tree is a.s. finite and no conditions on the $\left\{C_{i}\right\}$ are necessary for $R<\infty$ a.s. This corresponds to the second condition in Theorem 1 of [12]. (ii) In view of the same theorem from [12], one could possibly establish the convergence of $R_{n}^{*} \Rightarrow R<\infty$ under milder conditions. However, since in this paper we only study the power tails of $R$, the assumptions of Lemma 6 are not restrictive. (iii) Note that if $E\left[\sum_{i=1}^{N} C_{i}^{\alpha}\right]=1$ with $\alpha \in(0,1]$, then there might not be a $0<\beta<\alpha$ for which $E\left[\sum_{i=1}^{N} C_{i}^{\beta}\right]<1$, e.g., the case of deterministic $C_{i}$ 's that was studied in [42].

\subsection{The case when the weights $\left\{C_{i}\right\}$ dominate}

In this section we characterize the tail behavior of the distribution of the solution $R$ to the nonhomogeneous equation (1), as defined by (13), when its power-law tail behavior is due to the multiplicative effect of the weights $\left\{C_{i}\right\}$. The main result is given in the following theorem, which is an application of Theorem 1; see the proof of Theorem 4.1 in [27] and the remark at the end of this subsection. A generalization to real-valued weights can be found in Theorem 4.6 in [28].

Theorem 2. Let $\left(Q, N, C_{1}, C_{2}, \ldots\right)$ be a nonnegative random vector, with $N \in \mathbb{N} \cup$ $\{\infty\}, P(Q>0)>0$, and let $R$ be the solution to (1) given by (13). Suppose that there exists $j \geq 1$ with $P\left(N \geq j, C_{j}>0\right)>0$ such that the measure $P\left(\log C_{j} \in d u, C_{j}>0\right.$, $N \geq j)$ is nonarithmetic, and that for some $\alpha>0,0<E\left[\sum_{i=1}^{N} C_{i}^{\alpha} \log C_{i}\right]<\infty$, $E\left[\sum_{i=1}^{N} C_{i}^{\alpha}\right]=1$, and $E\left[Q^{\alpha}\right]<\infty$. In addition, assume 
1. $E\left[\sum_{i=1}^{N} C_{i}\right]<1$ and $E\left[\left(\sum_{i=1}^{N} C_{i}\right)^{\alpha}\right]<\infty$, if $\alpha>1$; or,
2. $E\left[\left(\sum_{i=1}^{N} C_{i}^{\alpha /(1+\varepsilon)}\right)^{1+\varepsilon}\right]<\infty$ for some $0<\varepsilon<1$, if $0<\alpha \leq 1$.

Then,

$$
P(R>t) \sim H t^{-\alpha}, \quad t \rightarrow \infty,
$$

where $0 \leq H<\infty$ is given by

$$
\begin{aligned}
H & =\frac{1}{E\left[\sum_{i=1}^{N} C_{i}^{\alpha} \log C_{i}\right]} \int_{0}^{\infty} v^{\alpha-1}\left(P(R>v)-E\left[\sum_{i=1}^{N} 1\left(C_{i} R>v\right)\right]\right) d v \\
& =\frac{E\left[\left(\sum_{i=1}^{N} C_{i} R_{i}+Q\right)^{\alpha}-\sum_{i=1}^{N}\left(C_{i} R_{i}\right)^{\alpha}\right]}{\alpha E\left[\sum_{i=1}^{N} C_{i}^{\alpha} \log C_{i}\right]} .
\end{aligned}
$$

Remark 6. (i) The nonhomogeneous equation has been previously studied for the special case when $Q$ and the $\left\{C_{i}\right\}$ are deterministic constants. In particular, Theorem 5 of [42] analyzes the solutions to (1) when $Q$ and the $\left\{C_{i}\right\}$ are nonnegative deterministic constants, which, when $\sum_{i=1}^{N} C_{i}^{\alpha}=1, \alpha>0$, implies that $C_{i} \leq 1$ for all $i$ and $\sum_{i} C_{i}^{\alpha} \log C_{i} \leq 0$, falling outside of the scope of this theorem. As previously mentioned, the additional stable-law solutions found recently in [5] for $Q$ and $\left\{C_{i}\right\}$ random also fall outside of the scope of this theorem and do not satisfy the conditions of Lemma 6. (ii) When $\alpha>1$, the condition $E\left[\left(\sum_{i=1}^{N} C_{i}\right)^{\alpha}\right]<\infty$ is needed to ensure that the tail of $R$ is not dominated by $\sum_{i=1}^{N} C_{i}$. In particular, if the $\left\{C_{i}\right\}$ are iid and independent of $N$, the condition reduces to $E\left[N^{\alpha}\right]<\infty$ since $E\left[C^{\alpha}\right]<\infty$ is implied by the other conditions; see Theorems 4.2 and 5.4 in [26]. Furthermore, when $0<\alpha \leq 1$ the condition $E\left[\left(\sum_{i=1}^{N} C_{i}\right)^{\alpha}\right]<\infty$ is redundant since $E\left[\left(\sum_{i=1}^{N} C_{i}\right)^{\alpha}\right] \leq E\left[\sum_{i=1}^{N} C_{i}^{\alpha}\right]=1$, and the additional condition $E\left[\left(\sum_{i=1}^{N} C_{i}^{\alpha /(1+\varepsilon)}\right)^{1+\varepsilon}\right]<\infty$ is needed. When the $\left\{C_{i}\right\}$ are iid and independent of $N$, the latter condition reduces to $E\left[N^{1+\varepsilon}\right]<\infty$ (given the other assumptions), which is consistent with Theorem 4.2 in [26]. (iii) Note that the second expression for $H$ is more suitable for actually computing it, especially in the case of $\alpha$ being an integer, as will be stated in the forthcoming Corollary 1, after which we will also explain how Lemma 2 can be used to derive an explicit upper bound on $H$ when $\alpha>1$ is not an integer. Regarding the lower bound, the elementary inequality $\left(\sum_{i=1}^{k} x_{i}\right)^{\alpha} \geq \sum_{i=1}^{k} x_{i}^{\alpha}$ for $\alpha \geq 1$ and $x_{i} \geq 0$, yields

$$
H \geq \frac{E\left[Q^{\alpha}\right]}{\alpha E\left[\sum_{i=1}^{N} C_{i}^{\alpha} \log C_{i}\right]}>0 .
$$

Similarly, for $0<\alpha<1$, using the corresponding inequality $\left(\sum_{i=1}^{k} x_{i}\right)^{\alpha} \leq \sum_{i=1}^{k} x_{i}^{\alpha}$ for $0<\alpha \leq 1, x_{i} \geq 0$, we obtain $H \leq E\left[Q^{\alpha}\right] /\left(\alpha E\left[\sum_{i=1}^{N} C_{i}^{\alpha} \log C_{i}\right]\right)$. (iv) Let us also observe that the solution $R$, given by (13), to equation (1) may be a constant 
(non power law) $R=r>0$ when $P\left(r=Q+r \sum_{i=1}^{N} C_{i}\right)=1$. However, similarly as in remark (i), such a solution is excluded from the theorem since $P(r=Q+$ $\left.r \sum_{i=1}^{N} C_{i}\right)=1$ implies $E\left[\sum_{i} C_{i}^{\alpha} \log C_{i}\right] \leq 0, \alpha>0$. (iv) The strict positivity of the constant $H$ for the real-valued case has very recently been established in [3], and a version where the weights $\left\{C_{i}\right\}$ are positive matrices and $Q$ is a positive vector can be found in [35].

As indicated earlier, when $\alpha \geq 1$ is an integer, we can obtain the following explicit expression for $H$.

Corollary 1. For integer $\alpha \geq 1$, and under the same assumptions of Theorem 2, the constant $H$ can be explicitly computed as a function of $E\left[R^{k}\right], 0 \leq k \leq \alpha-1$, and the mixed moments of order up to $\alpha$ of $\left(Q, N, C_{1}, C_{2}, \ldots\right)$ according to the following expression

$$
H=\frac{1}{\alpha E\left[\sum_{i=1}^{N} C_{i}^{\alpha} \log C_{i}\right]} E\left[Q^{\alpha}+\sum_{\mathbf{j} \in B_{\alpha-1}(N)}\left(\begin{array}{c}
\alpha \\
j_{0}, j_{1}, j_{2}, \ldots
\end{array}\right) Q^{j_{0}} \prod_{i=1}^{N} C_{i}^{j_{i}} E\left[R^{j_{i}}\right]\right],
$$

where $\mathbf{j}=\left(j_{0}, j_{1}, j_{2}, \ldots\right), B_{p}(n)=\left\{\left(j_{0}, j_{1}, j_{2}, \ldots\right) \in \mathbb{N}_{+}^{n+1}: \sum_{k=0}^{n} j_{k}=p, 0 \leq j_{i}<\right.$ $p$ \}. In particular, for $\alpha=1$,

$$
H=\frac{E[Q]}{E\left[\sum_{i=1}^{N} C_{i} \log C_{i}\right]},
$$

and for $\alpha=2$,

$$
\begin{aligned}
& H=\frac{E\left[Q^{2}\right]+2 E[R] E\left[Q \sum_{i=1}^{N} C_{i}\right]+2(E[R])^{2} E\left[\sum_{i=1}^{N} \sum_{j=i+1}^{N} C_{i} C_{j}\right]}{2 E\left[\sum_{i=1}^{N} C_{i}^{2} \log C_{i}\right]} \\
& E[R]=\frac{E[Q]}{1-E\left[\sum_{i=1}^{N} C_{i}\right]} .
\end{aligned}
$$

Proof. Using the multinomial expansion we obtain for any $k \in \mathbb{N}$

$$
\begin{aligned}
& E\left[\left(\sum_{i=1}^{N} C_{i} R_{i}+Q\right)^{\alpha}-\sum_{i=1}^{N}\left(C_{i} R_{i}\right)^{\alpha}\right] \\
& =E\left[Q^{\alpha}+\sum_{\mathbf{j} \in B_{\alpha-1}(N)}\left(\begin{array}{c}
\alpha \\
j_{0}, j_{1}, j_{2}, \ldots
\end{array}\right) Q^{j_{0}} \prod_{i=1}^{N}\left(C_{i} R_{i}\right)^{j_{i}}\right] .
\end{aligned}
$$

Next, condition on $\mathscr{F}=\sigma\left(Q, N, C_{1}, C_{2}, \ldots\right)$ to obtain 


$$
\begin{aligned}
& E\left[\sum_{\mathbf{j} \in B_{\alpha-1}(N)}\left(\begin{array}{c}
\alpha \\
j_{0}, j_{1}, j_{2}, \ldots
\end{array}\right) Q^{j_{0}} \prod_{i=1}^{N}\left(C_{i} R_{i}\right)^{j_{i}}\right] \\
& =E\left[\sum_{\mathbf{j} \in B_{\alpha-1}(N)}\left(\begin{array}{c}
\alpha \\
j_{0}, j_{1}, j_{2}, \ldots
\end{array}\right) Q^{j_{0}} \prod_{i=1}^{N} C_{i}^{j_{i}} E\left[R_{i}^{j_{i}} \mid \mathscr{F}\right]\right. \\
& =E\left[\sum_{\mathbf{j} \in B_{\alpha-1}(N)}\left(\begin{array}{c}
\alpha \\
j_{0}, j_{1}, j_{2}, \ldots
\end{array}\right) Q^{j_{0}} \prod_{i=1}^{N} C_{i}^{j_{i}} E\left[R^{j_{i}}\right]\right] .
\end{aligned}
$$

For the case when $\alpha>1$ is not an integer, the same arguments used in the proof of Lemma 2 (see Lemma 4.1 in [27]) lead to

$$
\begin{aligned}
& E\left[\left(\sum_{i=1}^{N} C_{i} R_{i}+Q\right)^{\alpha}-\sum_{i=1}^{N}\left(C_{i} R_{i}\right)^{\alpha}\right] \\
& \leq E\left[Q^{\alpha}+\left(\sum_{\mathbf{j} \in B_{p}(N)}\left(\begin{array}{c}
p \\
j_{0}, j_{1}, j_{2}, \ldots
\end{array}\right) Q^{j_{0}} \prod_{i=1}^{N}\left(C_{i} R_{i}\right)^{j_{i}}\right)^{\alpha / p}\right]
\end{aligned}
$$

where $\mathbf{j}$ and $B_{p}(n)$ are defined as in Corollary 1 and $p=\lceil\alpha\rceil$. Then condition on $\mathscr{F}=\sigma\left(Q, N, C_{1}, C_{2}, \ldots\right)$ and use Jensen's inequality to obtain

$H \leq \frac{1}{\alpha E\left[\sum_{i=1}^{N} C_{i}^{\alpha} \log C_{i}\right]} E\left[Q^{\alpha}+\left(\sum_{\mathbf{j} \in B_{p}(N)}\left(\begin{array}{c}p \\ j_{0}, j_{1}, j_{2}, \ldots\end{array}\right) Q^{j_{0}} \prod_{i=1}^{N} C_{i}^{j_{i}} E\left[R^{j_{i}}\right]\right)^{\alpha / p}\right]$.

Note that the moments $E\left[R^{j_{i}}\right], j_{i} \leq\lceil\alpha\rceil-1$, can be computed recursively as in Corollary 1 . For the case $N \equiv 1$ the recent work in [16] provides a computable expression for $H$.

Two results that facilitate the verification of the conditions in Theorem 1 are given below in Lemmas 7 and 8 (Lemmas 4.6 and 4.7 in [27]). The first of these results transforms the integral conditions in Theorem 1 into an expression that can be verified by using the specific recursion being analyzed. These lemmas can be directly applied to analyze other max-plus recursions as well, such as those mentioned in (5). We illustrate the use of these results by giving a heuristic proof of Theorem 2 at the end of this section.

Lemma 7. Suppose $\left(N, C_{1}, C_{2}, \ldots\right)$ is a nonnegative random vector, with $N \in \mathbb{N} \cup$ $\{\infty\}$ and let $\left\{R_{i}\right\}_{i \in \mathbb{N}}$ be a sequence of iid nonnegative random variables independent of $\left(N, C_{1}, C_{2}, \ldots\right)$ having the same distribution as $R$. For $\alpha>0$, suppose that $\sum_{i=1}^{N}\left(C_{i} R_{i}\right)^{\alpha}<\infty$ a.s. and $E\left[R^{\beta}\right]<\infty$ for any $0<\beta<\alpha$. Furthermore, assume that $E\left[\left(\sum_{i=1}^{N} C_{i}^{\alpha /(1+\varepsilon)}\right)^{1+\varepsilon}\right]<\infty$ for some $0<\varepsilon<1$. Then, 


$$
\begin{aligned}
0 & \leq \int_{0}^{\infty}\left(E\left[\sum_{i=1}^{N} 1\left(C_{i} R_{i}>t\right)\right]-P\left(\max _{1 \leq i \leq N} C_{i} R_{i}>t\right)\right) t^{\alpha-1} d t \\
& =\frac{1}{\alpha} E\left[\sum_{i=1}^{N}\left(C_{i} R_{i}\right)^{\alpha}-\left(\max _{1 \leq i \leq N} C_{i} R_{i}\right)^{\alpha}\right]<\infty
\end{aligned}
$$

Lemma 8. Let $\left(Q, N, C_{1}, C_{2}, \ldots\right)$ be a nonnegative vector with $N \in \mathbb{N} \cup\{\infty\}$ and let $\left\{R_{i}\right\}$ be a sequence of iid random variables, independent of $\left(Q, N, C_{1}, C_{2}, \ldots\right)$. Suppose that for some $\alpha>1$ we have $E\left[Q^{\alpha}\right]<\infty, E\left[\left(\sum_{i=1}^{N} C_{i}\right)^{\alpha}\right]<\infty, E\left[R^{\beta}\right]<\infty$ for any $0<\beta<\alpha$, and $\sum_{i=1}^{N} C_{i} R_{i}<\infty$ a.s. Then

$$
E\left[\left(\sum_{i=1}^{N} C_{i} R_{i}+Q\right)^{\alpha}-\sum_{i=1}^{N}\left(C_{i} R_{i}\right)^{\alpha}\right]<\infty .
$$

We now give some of the key high-level heuristics behind the proof of Theorem 2. The first technical condition to check is that $E\left[R^{\beta}\right]<\infty$ for all $0<\beta<\alpha$, which follows from the assumptions in the theorem and our moment estimates from Lemmas 3 and 4. The majority of the work in the proof goes into verifying the absolute integrability condition (7). In order to do this, we first observe that

$$
P(R>t)=P\left(\sum_{i=1}^{N} C_{i} R_{i}+Q>t\right) \approx P\left(\sum_{i=1}^{N} C_{i} R_{i}>t\right)
$$

for large $t$ since $E\left[Q^{\alpha}\right]<\infty$ and the weighted sum $\sum C_{i} R_{i}$ is expected to have infinite $\alpha$-moment. Then, under the condition $E\left[\left(\sum_{i=1}^{N} C_{i}\right)^{\alpha}\right]<\infty(\alpha>1)$, we expect the weighted sum to behave as the maximum according to the well-known heavy-tailed one-big-jump principle, i.e.,

$$
P\left(\sum_{i=1}^{N} C_{i} R_{i}>t\right) \approx P\left(\max _{1 \leq i \leq N} C_{i} R_{i}>t\right) .
$$

The last observation is that

$$
P\left(\max _{1 \leq i \leq N} C_{i} R_{i}>t\right) \approx E\left[\sum_{i=1}^{N} 1\left(C_{i} R_{i}>t\right)\right],
$$

for large $t$, which is made rigorous in Lemma 7. Hence, the proof is enabled by adding and subtracting the term $P\left(\max _{1 \leq i \leq N} C_{i} R_{i}>t\right)$ inside the integrand in (7). The rigorous justification of these ideas is quite involved, in part because one has to understand the second order properties of the preceding approximations, i.e., the error term; we refer the reader to $[26,27,28]$ for the details. 


\subsection{The case when the sum of the weights $\sum_{i=1}^{N} C_{i}$ dominates}

In this section we focus on the case where $P\left(Z_{N}>x\right) \in \mathscr{R}_{-\alpha}$ for some $\alpha>1$, and $\rho \vee \rho_{\alpha}<1$. The approach we follow is to first describe the asymptotic behavior of finitely many iterations of (1), those given by $R^{(n)}$, and then use the uniform bound given in Proposition 1 to control the difference $\left|R-R^{(n)}\right|$. The first lemma given below is based on the use of some asymptotic limits for randomly stopped and randomly weighted sums recently developed in [37].

Lemma 9. Let $Z_{N}=\sum_{i=1}^{N} C_{i}$ and suppose $P\left(Z_{N}>x\right) \in \mathscr{R}_{-\alpha}$ with $\alpha>1, E\left[Q^{\alpha+\varepsilon}\right]<$ $\infty, \rho_{\alpha+\varepsilon}<\infty$ for some $\varepsilon>0$, and $\rho<1$. Then, for any fixed $n \in\{1,2,3, \ldots\}$,

$$
P\left(R^{(n)}>x\right) \sim \frac{(E[Q])^{\alpha}}{(1-\rho)^{\alpha}} \sum_{k=0}^{n-1} \rho_{\alpha}^{k}\left(1-\rho^{n-k}\right)^{\alpha} P\left(Z_{N}>x\right)
$$

as $x \rightarrow \infty$, where $R^{(n)}$ was defined in (11).

Remark 7. In terms of the ranking example given in the introduction, $Q$ usually refers to a nonnegative personalization parameter that determines what page to go to in case the algorithm reaches a page with no outbound links (see [47] for more details).

From Lemma 9 one can already guess that, provided $\rho \vee \rho_{\alpha}<1$, the tail behavior of $R$ will be

$$
P(R>x) \sim \frac{(E[Q])^{\alpha}}{(1-\rho)^{\alpha}} \sum_{k=0}^{\infty} \rho_{\alpha}^{k} P\left(Z_{N}>x\right)
$$

as $x \rightarrow \infty$, assuming that the exchange of limits is justified. As mentioned above, this exchange represents the main technical difficulty. This result was proved in [26] for the case where $Q, N,\left\{C_{i}\right\}$ are all independent and the $\left\{C_{i}\right\}$ are iid using sample-path arguments, and in [47] for the case where $(Q, N)$ is independent of $\left\{C_{i}\right\}$ and the $\left\{C_{i}\right\}$ are iid, using transform methods and Tauberian theorems. A version of the results presented here that can be applied when $Q$ is a real-valued random variable can be found in [36].

The uniform bound given by Proposition 1 is the key to establishing that $\mid R-$ $R^{(n)} \mid$ goes to zero geometrically fast, which is more precisely stated in the following lemma.

Lemma 10. Let $Z_{N}=\sum_{i=1}^{N} C_{i}$ and suppose $P\left(Z_{N}>x\right) \in \mathscr{R}_{-\alpha}$ with $\alpha>1, E\left[Q^{\alpha+\varepsilon}\right]<$ $\infty$ and $\rho_{\alpha+\varepsilon}<\infty$, for some $\varepsilon>0$. Assume $\rho \vee \rho_{\alpha}<1$, then, for any fixed $0<\delta<1$, $n_{0} \in\{1,2, \ldots\}$ and $\rho \vee \rho_{\alpha}<\eta<1$, there exists a finite constant $K>0$ that does not depend on $\delta$ or $n_{0}$ such that

$$
\lim _{x \rightarrow \infty} \frac{P\left(\left|R-R^{\left(n_{0}\right)}\right|>\delta x\right)}{P\left(Z_{N}>x\right)} \leq \frac{K \eta^{n_{0}+1}}{\delta^{\alpha+1} n_{0}} .
$$


Combining Lemma 9 and Lemma 10 one can obtain the following result. The proofs of all the results in this subsection can be found in [36].

Theorem 3. Let $Z_{N}=\sum_{i=1}^{N} C_{i}$ and suppose $P\left(Z_{N}>x\right) \in \mathscr{R}_{-\alpha}$ with $\alpha>1, E\left[Q^{\alpha+\varepsilon}\right]<\infty$ and $\rho_{\alpha+\varepsilon}<\infty$, for some $\varepsilon>0$. Assume $\rho \vee \rho_{\alpha}<1$, then,

$$
P(R>x) \sim \frac{(E[Q])^{\alpha}}{(1-\rho)^{\alpha}\left(1-\rho_{\alpha}\right)} P\left(Z_{N}>x\right)
$$

as $x \rightarrow \infty$, where $R$ was defined in (13).

Remark 8. (i) For the case where the $\left\{C_{i}\right\}$ are iid and independent of $N$, and $P(N>$ $x) \in \mathscr{R}_{-\alpha}$, Lemma 3.7(2) in [29] gives

$$
P\left(Z_{N}>x\right) \sim\left(E\left[C_{1}\right]\right)^{\alpha} P(N>x) \quad \text { as } x \rightarrow \infty .
$$

(ii) Given the previous remark, it follows that Theorem 3 generalizes both Theorem 5.1 in [26] (for $Q, N,\left\{C_{i}\right\}$ all independent and $\left\{C_{i}\right\}$ iid) and the corresponding result from Section 3.4 in [47] (for $(Q, N)$ independent of $\left\{C_{i}\right\},\left\{C_{i}\right\}$ iid, $E[Q]<1$ and $E[C]=(1-E[Q]) / E[N])$. (iii) In view of Lemma 9, the theorem shows that the limits $\lim _{x \rightarrow \infty} \lim _{n \rightarrow \infty} P\left(R^{(n)}>x\right) / P\left(Z_{N}>x\right)$ are interchangeable.

\subsection{The case when $Q$ dominates}

This section of the paper treats the case where the heavy-tailed behavior of $R$ arises from the $\left\{Q_{\mathbf{i}}\right\}$, known in the autoregressive processes literature as innovations. This setting is well known in the special case $N \equiv 1$, since then the linear fixed-point equation (1) reduces to

$$
R \stackrel{\mathscr{D}}{=} C R+Q,
$$

where $(C, Q)$ are generally dependent. This fixed-point equation is the one satisfied by the steady state of the autoregressive process of order one with random coefficients, $\operatorname{RCA}(1)$ (see $[31,12,19,21])$.

That the innovations $\left\{Q_{\mathbf{i}}\right\}$ can give rise to heavy tails when the $\alpha$ mentioned above does not exist is also well known, see, e.g., [21, 32]; the main theorem of this subsection provides an alternative derivation of the forward implication in Theorem 1 from [21] (see also Proposition 2.4 in [32]) for $Q, N \geq 0$.

The results presented here are very similar to those in Subsection 5.2, and so are their proofs, which can also be found in [36] and include the case where $Q$ is real-valued.

Lemma 11. Suppose $P(Q>x) \in \mathscr{R}_{-\alpha}$, with $\alpha>1$, and $E\left[Z_{N}^{\alpha+\varepsilon}\right]<\infty$, for some $\varepsilon>0$. Then, for any fixed $n \in\{1,2,3, \ldots\}$,

$$
P\left(R^{(n)}>x\right) \sim \sum_{k=0}^{n} \rho_{\alpha}^{k} P(Q>x)
$$


as $x \rightarrow \infty$, where $R^{(n)}$ was defined in (13).

As for the case when $Z_{N}=\sum_{i=1}^{N} C_{i}$ dominates the asymptotic behavior of $R$, we can expect that,

$$
P(R>x) \sim\left(1-\rho_{\alpha}\right)^{-1} P(Q>x),
$$

and the technical difficulty is in justifying the exchange of the limits. The same techniques used in Subsection 5.2 can be used in this case as well. We point out that even though the condition $\rho<1$ is not necessary for the proportionality constant in Lemma 11 to be finite, it is required for the finiteness of $E[R]$.

The corresponding version of Lemma 10 is given below.

Lemma 12. Let $Z_{N}=\sum_{i=1}^{N} C_{i}$ and suppose $P(Q>x) \in \mathscr{R}_{-\alpha}$ with $\alpha>1, E\left[Z_{N}^{\alpha+\varepsilon}\right]<$ $\infty$ for some $\varepsilon>0$, and $E\left[Q^{\beta}\right]<\infty$ for all $0<\beta<\alpha$. Assume $\rho \vee \rho_{\alpha}<1$, then, for any fixed $0<\delta<1, n_{0} \in\{1,2, \ldots\}$ and $\rho \vee \rho_{\alpha}<\eta<1$, there exists a finite constant $K>0$ that does not depend on $\delta$ or $n_{0}$ such that

$$
\lim _{x \rightarrow \infty} \frac{P\left(\left|R-R^{\left(n_{0}\right)}\right|>\delta x\right)}{P(Q>x)} \leq \frac{K \eta^{n_{0}+1}}{\delta^{\alpha+1} n_{0}} .
$$

The main theorem of this section is given below.

Theorem 4. Suppose $P(Q>x) \in \mathscr{R}_{-\alpha}$, with $\alpha>1, E\left[Q^{\beta}\right]<\infty$ for all $0<\beta<\alpha$. Assume $\rho \vee \rho_{\alpha}<1$, and $E\left[Z_{N}^{\alpha+\varepsilon}\right]<\infty$ for some $\varepsilon>0$. Then,

$$
P(R>x) \sim\left(1-\rho_{\alpha}\right)^{-1} P(Q>x)
$$

as $x \rightarrow \infty$, where $R$ was defined in (13).

Remark 9. (i) This result generalizes Theorem 1 in [21] for the case $N \equiv 1$ (the forward implication, $\alpha>1$ ) to the weighted branching tree when $Q \geq 0$. It also generalizes the results in $[47,26]$ in the same way as Theorem 3 does for the case where $Z_{N}$ dominates. (ii) It is also worth pointing out that the same sample-path techniques used here can be used to study the intermediate case where $P(Q>x) \sim$ $K P\left(Z_{N}>x\right)$ for some constant $K>0$, which is also analyzed in [47] under stronger conditions than those stated above.

\section{Other recursions}

In this section we show how our techniques can be applied to study other recursions on trees, e.g., those stated in (5). In particular, we start with the following non-linear equation

$$
R \stackrel{\mathscr{D}}{=}\left(\bigvee_{i=1}^{N} C_{i} R_{i}\right) \vee Q
$$


where $\left(Q, N, C_{1}, C_{2}, \ldots\right)$ is a nonnegative random vector with $N \in \mathbb{N} \cup\{\infty\}, P(Q>$ $0)>0$ and $\left\{R_{i}\right\}_{i \in \mathbb{N}}$ is a sequence of iid random variables that have the same distribution as $R$ and is independent of $\left(Q, N, C_{1}, C_{2}, \ldots\right)$. Note that in the case of page ranking applications, where the $\left\{R_{i}\right\}$ represent the ranks of the neighboring pages, the potential ranking algorithm defined by the preceding recursion, determines the rank of a page as a weighted version of the most highly ranked neighboring page. In other words, the highest ranked reference has the dominant impact. Similarly to the homogeneous linear case, this recursion was previously studied in [7] under the assumption that $Q \equiv 0, N=\infty$, and the $\left\{C_{i}\right\}$ are real-valued deterministic constants. The more closely related case of $Q \equiv 0$ and $\left\{C_{i}\right\} \geq 0$ being random was studied earlier in [25].

Using standard arguments, we start by constructing a solution to (17) on a tree and then we show that this solution is finite a.s. and unique under iterations (under some moment conditions), similarly to what was done for the nonhomogeneous linear recursion in Section 5. Our main result of this section is stated in Theorem 5.

Following the same notation as in Section 5, define the process

$$
V_{n}=\bigvee_{\mathbf{i} \in A_{n}} Q_{\mathbf{i}} \Pi_{\mathbf{i}}, \quad n \geq 0,
$$

on the weighted branching tree $\mathscr{T}_{Q, C}$, as constructed in Section 2. Recall that the convention is that $\left(Q, N, C_{1}, C_{2}, \ldots\right)=\left(Q_{\emptyset}, N_{\emptyset}, C_{(\emptyset, 1)}, C_{(\emptyset, 2)}, \ldots\right)$ denotes the random vector corresponding to the root node.

With a possible abuse of notation relative to Section 5, define the process $\left\{R^{(n)}\right\}_{n \geq 0}$ according to

$$
R^{(n)}=\bigvee_{k=0}^{n} V_{k}, \quad n \geq 0
$$

Just as with the linear recursion from Section 5, it is not hard to see that $R^{(n)}$ satisfies the recursion

$$
R^{(n)}=\left(\bigvee_{j=1}^{N_{\emptyset}} C_{(\emptyset, j)} R_{j}^{(n-1)}\right) \vee Q_{\emptyset}=\left(\bigvee_{j=1}^{N} C_{j} R_{j}^{(n-1)}\right) \vee Q,
$$

where $\left\{R_{j}^{(n-1)}\right\}$ are independent copies of $R^{(n-1)}$ corresponding to the subtree starting with individual $j$ in the first generation and ending on the $n$th generation. One can also verify that

$$
V_{n}=\bigvee_{k=1}^{N_{\emptyset}} C_{(\emptyset, k)} \bigvee_{\left(k, \ldots, i_{n}\right) \in A_{n}} Q_{\left(k, \ldots, i_{n}\right)} \prod_{j=2}^{n} C_{\left(k, \ldots, i_{j}\right)} \stackrel{\mathscr{D}}{=} \bigvee_{k=1}^{N} C_{k} V_{(n-1), k}
$$

where $\left\{V_{(n-1), k}\right\}$ is a sequence of iid random variables independent of $\left(N, C_{1}, C_{2}, \ldots\right)$ and having the same distribution as $V_{n-1}$.

We now define the random variable $R$ according to 


$$
R \triangleq \lim _{n \rightarrow \infty} R^{(n)}=\bigvee_{k=0}^{\infty} V_{k}
$$

Note that $R^{(n)}$ is monotone increasing sample-pathwise, so $R$ is well defined. Also, by monotonicity of $R^{(n)},(19)$ and monotone convergence, we obtain that $R$ solves

$$
R=\left(\bigvee_{j=1}^{N_{\emptyset}} C_{(\emptyset, j)} R_{j}^{(\infty)}\right) \vee Q_{\emptyset}=\left(\bigvee_{j=1}^{N} C_{j} R_{j}^{(\infty)}\right) \vee Q
$$

where $\left\{R_{j}^{(\infty)}\right\}_{j \in \mathbb{N}}$ are iid copies of $R$, independent of $\left(Q, N, C_{1}, C_{2}, \ldots\right)$. Clearly this implies that $R$, as defined by (20), is a solution in distribution to (17). However, this solution might be $\infty$. Now, we establish the finiteness of moments of $R$, and in particular that $R<\infty$ a.s., in the following lemma.

Lemma 13. Assume that $\rho_{\beta}=E\left[\sum_{i=1}^{N} C_{i}^{\beta}\right]<1$ and $E\left[Q^{\beta}\right]<\infty$ for some $\beta>0$. Then, $E\left[R^{\gamma}\right]<\infty$ for all $0<\gamma \leq \beta$, and in particular, $R<\infty$ a.s. Moreover, if $\beta \geq 1$, $R^{(n)} \stackrel{L_{\beta}}{\rightarrow} R$, where $L_{\beta}$ stands for convergence in $\left(E|\cdot|^{\beta}\right)^{1 / \beta}$ norm.

Just as with the linear recursion from Section 5, we can define the process $\left\{R_{n}^{*}\right\}$ as

$$
R_{n}^{*} \triangleq R^{(n-1)} \vee V_{n}\left(R_{0}^{*}\right), \quad n \geq 1
$$

where

$$
V_{n}\left(R_{0}^{*}\right)=\bigvee_{\mathbf{i} \in A_{n}} R_{0, \mathbf{i}}^{*} \Pi_{\mathbf{i}}
$$

and $\left\{R_{0, \mathbf{i}}^{*}\right\}_{\mathbf{i} \in U}$ are iid copies of an initial value $R_{0}^{*}$, independent of the entire weighted tree $\mathscr{T}_{Q, C}$. It follows from (19) and (21) that

$$
R_{n+1}^{*}=\bigvee_{j=1}^{N} C_{j}\left(R_{j}^{(n-1)} \vee \bigvee_{\mathbf{i} \in A_{n, j}} R_{0, \mathbf{i}}^{*} \prod_{k=2}^{n} C_{\left(j, \ldots, i_{k}\right)}\right) \vee Q=\bigvee_{j=1}^{N} C_{j} R_{n, j}^{*} \vee Q,
$$

where $\left\{R_{j}^{(n-1)}\right\}$ are independent copies of $R^{(n-1)}$ corresponding to the subtree starting with individual $j$ in the first generation and ending on the $n$th generation, and $A_{n, j}$ is the set of all nodes in the $(n+1)$ th generation that are descendants of individual $j$ in the first generation. Moreover, $\left\{R_{n, j}^{*}\right\}$ are iid copies of $R_{n}^{*}$, and thus, $R_{n}^{*}$ is equal in distribution to the process obtained by iterating (17) with an initial condition $R_{0}^{*}$. This process can be shown to converge in distribution to $R$ for any initial condition $R_{0}^{*}$ satisfying the following moment condition.

Lemma 14. For any $R_{0}^{*} \geq 0$, if $E\left[Q^{\beta}\right], E\left[\left(R_{0}^{*}\right)^{\beta}\right]<\infty$ and $\rho_{\beta}<1$ for some $\beta>0$, then

$$
R_{n}^{*} \Rightarrow R
$$

with $E\left[R^{\beta}\right]<\infty$. Furthermore, under these assumptions, the distribution of $R$ is the unique solution with finite $\beta$-moment to recursion (17). 
We now state the main result of this section; see Theorem 5.1 in [27].

Theorem 5. Let $\left(Q, N, C_{1}, C_{2}, \ldots\right)$ be a nonnegative random vector, with $N \in \mathbb{N} \cup$ $\{\infty\}, P(Q>0)>0$ and $R$ be the solution to (17) given by (20). Suppose that there exists $j \geq 1$ with $P\left(N \geq j, C_{j}>0\right)>0$ such that the measure $P\left(\log C_{j} \in\right.$ $\left.d u, C_{j}>0, N \geq j\right)$ is nonarithmetic, and that for some $\alpha>0, E\left[Q^{\alpha}\right]<\infty, 0<$ $E\left[\sum_{i=1}^{N} C_{i}^{\alpha} \log C_{i}\right]<\infty$ and $E\left[\sum_{i=1}^{N} C_{i}^{\alpha}\right]=1$. In addition, assume

1. $E\left[\left(\sum_{i=1}^{N} C_{i}\right)^{\alpha}\right]<\infty$, if $\alpha>1$; or, 2. $E\left[\left(\sum_{i=1}^{N} C_{i}^{\alpha /(1+\varepsilon)}\right)^{1+\varepsilon}\right]<\infty$ for some $0<\varepsilon<1$, if $0<\alpha \leq 1$.

Then,

$$
P(R>t) \sim H t^{-\alpha}, \quad t \rightarrow \infty,
$$

where $0 \leq H<\infty$ is given by

$$
\begin{aligned}
H & =\frac{1}{E\left[\sum_{i=1}^{N} C_{i}^{\alpha} \log C_{i}\right]} \int_{0}^{\infty} v^{\alpha-1}\left(P(R>v)-E\left[\sum_{i=1}^{N} 1\left(C_{i} R>v\right)\right]\right) d v \\
& =\frac{E\left[\left(\bigvee_{i=1}^{N} C_{i} R_{i}\right)^{\alpha} \vee Q^{\alpha}-\sum_{i=1}^{N}\left(C_{i} R_{i}\right)^{\alpha}\right]}{\alpha E\left[\sum_{i=1}^{N} C_{i}^{\alpha} \log C_{i}\right]} .
\end{aligned}
$$

As an illustration of the generality of the developed techniques, we now discuss another example that is closely related to recursion (17), which is given by

$$
R \stackrel{\mathscr{D}}{=}\left(\bigvee_{i=1}^{N} C_{i} R_{i}\right)+Q
$$

where $\left(Q, N, C_{1}, C_{2}, \ldots\right)$ is a nonnegative vector with $N \in \mathbb{N} \cup\{\infty\}, P(Q>0)>0$, and $\left\{R_{i}\right\}_{i \in \mathbb{N}}$ is a sequence of iid random variables independent of $\left(Q, N, C_{1}, C_{2}, \ldots\right)$ having the same distribution as $R$. Its analysis could follow very closely the steps used for the linear and maximum recursions, except that the constructed solution $R$ would be less explicit. More specifically, one could iterate (22), similarly as it was done in (19) for the maximum recursion. To this end, an iteration $R^{(n)}$ could be constructed as a function of the weights of the first $n$ generations of the tree, and would solve

$$
R^{(n)}=\left(\bigvee_{j=1}^{N} C_{j} R_{j}^{(n-1)}\right)+Q,
$$

where $\left\{R_{j}^{(n-1)}\right\}$ is the corresponding iteration obtained on a subtree that starts on node $j$ in the first generation and ends on the $n$th generation; clearly $\left\{R_{j}^{(n-1)}\right\}$ is a sequence of iid random variables. Furthermore, it appears that $R^{(n)}$ is monotonically increasing in $n$, see equation (37) in [1], and thus its limit $R=R^{(\infty)}=\lim _{n \rightarrow \infty} R^{(n)}$ is properly defined. In addition, by using monotonicity arguments, one can show that 


$$
R^{(\infty)}=\left(\bigvee_{j=1}^{N} C_{j} R_{j}^{(\infty)}\right)+Q
$$

where $\left\{R_{j}^{(\infty)}\right\}$ is the corresponding iterative solution constructed on the infinite subtree that starts at node $j$ in the first generation. Hence, such a constructed $R$ is a solution to (22). Also, since $R$ is bounded from above by the solution (13) to the nonhomogeneous linear equation, sufficient conditions for the finiteness of its moments can be obtained from the corresponding results for the solution in (13). Recursion (22) was termed "discounted tree sums" in [1]; for additional details on the existence and uniqueness of its solution see Section 4.4 in [1].

Similarly one could study the third equation from (5),

$$
R \stackrel{\mathscr{D}}{=}\left(\sum_{i=1}^{N} C_{i} R_{i}\right) \vee Q
$$

by first constructing iteratively an endogenous solution on the weighted branching tree and then develop the conditions for the finiteness of its moments, etc.

Acknowledgements This work is supported by NSF Grant CMMI-1131053.

\section{References}

1. D.J. Aldous and A. Bandyopadhyay. A survey of max-type recursive distributional equation. Ann. Appl. Probab., 15(2):1047-1110, 2005.

2. G. Alsmeyer, J.D. Biggins, and M. Meiners. The functional equation of the smoothing transform. arXiv:0906.3133. To appear in Ann. Probab., 2012.

3. G. Alsmeyer, E. Damek, and S. Mentemeier. Tails of fixed points of the two-sided smoothing transform. Springer Proceedings in Mathematics \& Mathematics: Random Matrices and Iterated Random Functions. Springer-Verlag, 2012.

4. G. Alsmeyer and D. Kuhlbusch. Double martingale structure and existence of $\phi$-moments for weighted branching processes. Münster Journal of Mathematics, 1, 2008.

5. G. Alsmeyer and M. Meiners. Fixed points of the smoothing transform: Two-sided solutions. arXiv:1009.2412. To appear in Probab. Theory Relat. Fields, 2012.

6. G. Alsmeyer and U. Rösler. A stochastic fixed point equation related to weighted branching with deterministic weights. Electron. J. Probab., 11:27-56, 2005.

7. G. Alsmeyer and U. Rösler. A stochastic fixed point equation for weighted minima and maxima. Annales de l'Institiut Henri Poincaré, 44(1):89-103, 2008.

8. K.B. Athreya, D. McDonald, and P. Ney. Limit theorems for semi-Markov processes and renewal theory for Markov chains. Ann. Probab., 6(5):788-797, 1978.

9. Y. Baryshnikov and A. Gnedin Counting intervals in the packing process Ann. Appl. Probab., 11(3):863-877, 2001.

10. J.D. Biggins. Martingale convergence in the branching random walk. Journal Appl. Prob., 14(1):25-37, 1977.

11. J.D. Biggins and A.E. Kyprianou. Seneta-heyde norming in the branching random walk. Ann. Probab., 25(1):337-360, 1997.

12. A. Brandt. The stochastic equation $y_{n+1}=a_{n} y_{n}+b_{n}$ with stationary coefficients. Adv. Appl. Probab., 18(1):211-220, 1986. 
13. D. Buraczewski, E. Damek, S. Mentemeier and M. Mirek. Heavy tailed solutions of multivariate smoothing transforms. arXiv:12-6.1709, 2012.

14. Y.S. Chow and H. Teicher. Probability Theory. Springer-Verlag, New York, 1988.

15. E.G. Coffman Jr., L. Flatto, P.R. Jelenković and B. Poonen. Packing Random Intervals OnLine. Algorithmica, 22(4):448-476, 1998.

16. J.F. Collamore and A.N. Vidyashankar. Tail estimates for stochastic fixed point equations via nonlinear renewal theory. arXiv:1103.2317, 2011.

17. R. Durret and T. Liggett. Fixed points of the smoothing transformation. Z. Wahrsch. verw. Gebeite, 64:275-301, 1983.

18. J.A. Fill and S. Janson. Approximating the limiting Quicksort distribution. Random Structures Algorithms, 19(3-4):376-406, 2001.

19. C.M. Goldie. Implicit renewal theory and tails of solutions of random equations. Ann. Appl. Probab., 1(1):126-166, 1991.

20. C.M. Goldie and R. Grübel. Perpetuities with thin tails. Adv. Appl. Prob., 28(2):463-480, 1996.

21. D.R. Grey. Regular variation in the tail behaviour of solutions of random difference equations. Ann. Appl. Probab., 4(1):169-183, 1994.

22. A.K. Grincevičius. One limit distribution for a random walk on the line. Lithuanian Math. J., 15:580-589, 1975.

23. R. Holley and T. Liggett. Generalized potlatch and smoothing processes. Z. Wahrsch. verw. Gebeite, 55:165-195, 1981.

24. A.M. Iksanov. Elementary fixed points of the BRW smoothing transforms with infinite number of summands. Stochastic Process. Appl., 114:27-50, 2004.

25. P. Jagers and U. Rösler. Stochastic fixed points involving the maximum. In Mathematics and Computer Science III (M. Drmota, P. Flajolet, D. Gardy and B. Gittenberger, eds.), 325-338. Birkhäuser, Basel, 2004.

26. P.R. Jelenković and M. Olvera-Cravioto. Information ranking and power laws on trees. $A d v$. Appl. Prob., 42(4):1057-1093, 2010.

27. P.R. Jelenković and M. Olvera-Cravioto. Implicit renewal theory and power tails on trees. Adv. Appl. Prob., 44(2):528-561, 2012.

28. P.R. Jelenković and M. Olvera-Cravioto. Implicit renewal theory for trees with general weights. Stochastic Process. Appl.,122(9):3209-3238, 2012.

29. A.H. Jessen and T. Mikosch. Regularly varying functions. Publications de L'Institut Mathematique, Nouvelle Serie, 80(94):171-192, 2006.

30. J.P. Kahane and J. Peyrière. Sur certaines martingales de benoit mandelbrot. Adv. Math., 22:131-145, 1976.

31. H. Kesten. Random difference equations and renewal theory for products of random matrices. Acta Mathematica, 131(1):207-248, 1973.

32. D.G. Konstantinides and T. Mikosch. Large deviations and ruin probabilities for solutions to stochastic recurrence equations with heavy-tailed innovations. Ann. Probab., 33(5):1992$2035,2005$.

33. Q. Liu. Fixed points of a generalized smoothing transformation and applications to the branching random walk. Adv. Appl. Prob., 30:85-112, 1998.

34. Q. Liu. On generalized multiplicative cascades. Stochastic Process. Appl., 86:263-286, 2000.

35. M. Mirek. On fixed points of a generalized multidimensional affine recursion. arXiv:1111.1756v1, 2011.

36. M. Olvera-Cravioto. Tail behavior of solutions of linear recursions on trees. Stochastic Process. Appl., 122(4):1777-1807, 2012.

37. M. Olvera-Cravioto. Asymptotics for weighted random sums. Adv. Appl. Prob., 44(4), 2012.

38. P. Negadailov. Limit theorems for random recurrences and renewal-type processes. 2010. $\mathrm{PhD}$ Thesis. Available at http://igitur-archive.library.uu.nl/dissertations/.

39. R. Neininger On a multivariate contraction method for random recursive structures with applications to Quicksort. Random Structures Algorithms, 19:498-524, 2001.

40. R. Neininger and L. Rüschendorf. A general limit theorem for recursive algorithms and combinatorial structures. Ann. Appl. Prob., 14(1):378-418, 2004. 
41. U. Rösler. A limit theorem for Quicksort. RAIRO Inform. Thor. Appl., 25:85-100.

42. U. Rösler. The weighted branching process. Dynamics of complex and irregular systems (Bielefeld, 1991), pages 154-165, 1993. Bielefeld Encounters in Mathematics and Physics VIII, World Science Publishing, River Edge, NJ.

43. U. Rösler and L. Rüschendorf. The contraction method for recursive algorithms. Algorithmica, 29(1-2):3-33, 2001.

44. M.S. Sgibnev. The matrix analogue of the Blackwell renewal theorem on the real line. Sbornik: Mathematics, 197(3):369-386, 2006.

45. Y. Volkovich. Stochastic analysis of web page ranking. Ph.D. Thesis, University of Twente, 2009.

46. Y. Volkovich, N. Litvak, and D. Donato. Determining factors behind the pagerank log-log plot. Proceedings of the 5th International Workshop on Algorithms and Models for the WebGraph, WAW 2007, 2007.

47. Y. Volkovich and N. Litvak. Asymptotic analysis for personalized web search. Adv. Appl. Prob., 42(2):577-604, 2010.

48. E.C. Waymire and S.C. Williams. Multiplicative cascades: dimension spectra and dependence. J. Fourier Anal. Appl, pages 589-609, 1995. Kahane Special Issue. 